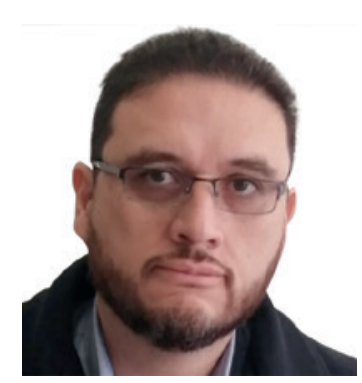

\title{
Hipertensión pulmonar: Correlación de los marcadores plasmáticos con la supervivencia de los pacientes
}

Marco Hugo Sánchez-Bustillos

Hospital Star Médica, Chihuahua, México

Resumen de Koudstaal T, van Uden D, van Hulst JAC, Heukels P, Bergen IM, Geenen LW, Baggen VJM, van den Bosch AE, van den Toorn LM, Chandoesing PP, Kool M, Boersma E, Hendriks RW, Boomars KA. Plasma markers in pulmonary hypertension subgroups correlate with patient survival. Respir Res. 2021;22(1):137.

\author{
Keywords \\ Pulmonary hypertension · Inflammation and immunity . \\ Inflammatory cytokines · Survival and prognosis · Biomarkers \\ Pulmonary arterial hypertension . Chronic thromboembolic \\ pulmonary hypertension
}

\begin{abstract}
Background: Recent studies have provided evidence for an important contribution of the immune system in the pathophysiology of pulmonary arterial hypertension $(\mathrm{PAH})$ and chronic thromboembolic pulmonary hypertension (CTEPH). In this report, we investigated whether the inflammatory profile of pulmonary hypertension patients changes over time and correlates with patient WHO subgroups or survival.
\end{abstract}

Methods: 50 PAH patients (16 idiopathic (I)PAH, 24 Connective Tissue Disease (CTD)-PAH and 10 Congenital Heart Disease (CHD)-PAH), $37 \mathrm{CTEPH}$ patients and 18 healthy controls (HCS) were included in the study. Plasma inflammatory markers at baseline and after 1-year follow-up were measured using ELISAs. Subsequently, correlations with hemodynamic parameters and survival were explored and data sets were subjected to unbiased multivariate analyses.

Results: At diagnosis, we found that plasma levels of interleukin- 6 (IL-6) and the chemokines (C-X3-C) motif legend CXCL9 and CXCL13 in CTD-PAH patients were significantly increased, compared with HCs. In idiopathic PAH patients the levels of tumor growth factor- $\beta$ (TGF $\beta$ ), IL-10 and CXCL9 were elevated, compared with HCs. The increased CXCL9 and IL-8 concentrations in CETPH patients correlated significantly with decreased survival, suggesting that CXCL9 and IL-8 may be prognostic markers. After one year of treatment, IL-10, CXCL13 and TGF $\beta$ levels changed significantly in the PAH subgroups and CTEPH patients. Unbiased multivariate analysis revealed clustering of $\mathrm{PH}$ patients based on inflammatory mediators and clinical parameters, but did not separate the WHO subgroups. Importantly, these multivariate analyses separated patients with $<3$ years and $>3$ years survival, in particular when inflammatory mediators were combined with clinical parameters.

Discussion: Our study revealed elevated plasma levels of inflammatory mediators in different PAH subgroups and CTEPH at baseline and at 1-year follow-up, whereby CXCL9 and IL-8 may prove to be prognostic markers for CTEPH patients. While this study is exploratory and hypothesis generating, our data indicate an important role for IL-8 and CXCL9 in CHD and CTEPH patients considering the increased plasma levels and the observed correlation with survival.

Conclusion: In conclusion, our studies identified an inflammatory signature that clustered $\mathrm{PH}$ patients into WHO classificationindependent subgroups that correlated with patient survival.

(c) 2021 Koudstaal, van Uden, van Hulst, Heukels, Bergen, Geenen, Baggen, van den Bosch, van den Toorn, Chandoesing, Kool, Boersma, Hendriks, Boomars 


\section{Transferencia en la práctica}

\section{Contexto del estudio}

La hipertensión pulmonar (HP) es una enfermedad de mal pronóstico heterogénea en su etiología, y que a pesar del surgimiento de nuevas terapéuticas el pronóstico sigue siendo muy malo, ha surgido una nueva clasificación la cual orienta los tratamientos vaso dilatadores únicamente al grupo 1. Este trabajo de Thomas Koudstaal y colegas explora el rol de la inflamación mediante determinación de citocinas específicas como marcador pronostico. Se incluyeron 16 pacientes con hipertensión pulmonar idiopática (HPI), 24 con hipertensión pulmonar asociada a enfermedades del tejido conjuntivo (HPAETC), 10 con hipertensión pulmonar asociada a cardiopatía congénita (HPCC), 37 con hipertensión pulmonar secundaria a enfermedad pulmonar tromboembólica crónico (HPSEPTC) y 18 controles sanos (CS); todos fueron diagnosticados mediante cateterismo cardiaco. Pacientes con HP mayores de 18 años con presión arterial pulmonar media (mPAP) $\geq 25 \mathrm{mmHg}$, una presión de cuña $\leq 15 \mathrm{mmHg}$ y una PVR $\geq 3 \mathrm{WU}$ medida por cateterismo cardíaco derecho, se descartó enfermedad pulmonar previa mediante pruebas de función respiratoria y valoración por cardiólogo y neumólogo.

\section{Resultados del estudio}

Se midieron citocinas inflamatorias y dio seguimiento para valorar sobrevida o trasplante, las citocinas fueron VEGFA, TGF $\beta$, CXCL-9, CXCL-13, IL-1ß, IL-6, IL-8, IL-10 semestralmente.

Al inicio se encontraron aumentados los niveles plasmáticos de IL10, TGF $\beta$ y CXCL9 en pacientes con HPI, de IL-6, CXCL9 y CXCL13 en pacientes con HPAETC, y de CXCL9 en pacientes con HPSEPTC, TGF $\beta$ estaba aumentado en los pacientes HPI y HPCC; todos en comparación con los pacientes del grupo control, así mismo no hubo diferencias entre los grupos y el control en cuanto a los marcadores hemodinámicos.

El promedio de seguimiento fue de 39.5 meses por paciente, al término del estudio 22 de 87 los pacientes habían muerto y 2 pacientes habían recibido un trasplante de pulmón.

En pacientes con HPCC y HPSEPTC que presentaban IL8 elevados al inicio del estudio se redujo su supervivencia en relación con los que lo tenían baja ( $p=0,013$ y $p=0,016$ ) respectivamente. Al comparar CXCL9 alto y CXCL9 bajo pacientes de estos mismos grupos también se redujo la sobrevida en el grupo con CXCL9 aumentado, ( $p=0,08$ y 0,01 respectivamente) sin significancia estadística para HPSEPTC.

Se evalúo también el tiempo para empeoramiento clínico (TECW), disminución del más del 15\% en la caminata de 6 minutos, el ingreso a hospital por causa del HP o necesidad de aumentar medica- ción especifica; sólo se encontró diferencia para en el grupo de HPSEPTC, con elevación de CXCL9 versus los de niveles bajos de este marcador.

En 31 pacientes se logró dar seguimiento durante un año completo se observó que para IL-13, IL-6, IL-8, CXCL-9 y VEGF, no se encontraron cambios significativos en ninguno de los grupos. Hubo un aumento significativo en IL-10 y TGF $\beta$ en el grupo HPAETC. Los pacientes con HPSEPTC mostraron una disminución significativa en los niveles de TGF $\beta y$ en los pacientes con HPI se mostró una disminución del CXCL-13.

Solo CXCL9 después de 1 año de seguimiento mostró una significativa correlación con la supervivencia en pacientes con HPSEPTC. No encontramos evidencia de una normalización de los marcadores inflamatorios al compararlos con los controles.

En base a estos hallazgos los investigadores proponen establecer perfiles inflamatorios en conjunto con parámetros hemodinámicos en pacientes con HPSEPTC y HPI, encontrando diferencias significativas al contar con diferencias en mPAP, PVR, CXCL9 y CXCL13.

\section{Conclusiones y recomendaciones para la práctica}

Este trabajo aporta un grupo significativo de pacientes tomando en cuenta la baja prevalencia de la hipertensión pulmonar. Se hace un análisis minucioso con gran fuerza estadística y por un tiempo prolongado. Los hallazgos más significativos son la relación de los niveles de IL8 y CXCL9 en relación con la sobrevida en los pacientes con HPSEPTC y HPCC los cuales tienen un significado pronostico ya que altos niveles de estos marcadores se asocian a una menor sobrevida. Llama la atención que en sus resultados no impacto el uso de fármacos específicos para HP.

Esto abre la posibilidad de nuevos blancos terapéuticos (que pasaría si logramos inhibir la IL 8 y el CXCL9) o ver en ellos un posible monitor de la efectividad de las terapéuticas instaladas, habrá que seguir los resultados de otros grupos sobre el tema.

Cabe destacar que si bien los otros marcadores no se correlacionaron con la disminución se la sobrevida, no se deben abandonar sino seguir el escrutinio sino hay que identificar cuál es su impacto individual o en conjunto sobre la enfermedad.

\section{Disclosure Statement}

Por la referencia el autor declara que no tiene conflicto de interés con respecto a esta transferencia de conocimientos.

Correspondencia:

Dr. Marco Hugo Sánchez-Bustillos, marcohugos@yahoo.com 